\title{
Induced Movement Based on Subtraction of Motion From the Inducing Object
}

\author{
Irvin Rock, Mitchell Auster, Mary Schiffman, and \\ Deborah Wheeler \\ Institute for Cognitive Studies, Rutgers - The State University
}

\begin{abstract}
Induced movement occurs not only when an object surrounding a stationary one moves below threshold but when it moves above threshold. The above-threshold effect is particularly puzzling and seemingly irrational because the relative displacement should be fully accounted for by perceiving the moving object in motion. The accepted theory has been that such excess motion can be explained in terms of a separation of systems: The enclosed object is governed by the relationship to its immediately surrounding frame of reference, and the latter is governed either by the relationship to its surrounding frame of reference or to the observer. An alternative explanation is that when induced motion occurs, the moving object is perceived to be either stationary or moving less than is warranted by its actual motion. Evidence is presented supporting this hypothesis according to which the relative displacement is apportioned phenomenally to either the induced object, the inducing object, or both. The objective motion of the inducing object is thus in whole or part transferred to the induced object. Thus, excess phenomenal motion does not occur, and induced movement can be regarded as the rational solution to a problem.
\end{abstract}

As with many phenomena of perception, induced movement has been studied experimentally over many decades, but there is little agreement as to its explanation. There is agreement that the displacement of one object relative to another (object-relative displacement) is an important source of information concerning motion. If one object is moving at a rate below threshold, the introduction of a second stationary object leads to an immediate impression of motion, and this impression must logically be based on relative displacement (Duncker, 1929; Wallach, 1959, 1976). Moreover, the stationary object will be seen in motion as often as the actually

This research was supported in part by National Institute of Mental Health Grant MH-30865.

The first author expresses his appreciation to Arien Mack, Frank Restle, and a group at York University, consisting of Stuart Anstis, Ian Howard, Hiroshi Ono, Martin Steinbach, and Brian Templeton for many helpful discussions of the problem of induced movement.

Requests for reprints should be sent to Irvin Rock, Institute for Cognitive Studies, Rutgers-The State University, Newark, New Jersey 07102. moving one (Mack, Fisher, \& Fendrich, 1975). If several objects are in motion, even above threshold, we know from Johansson's (1950) work that their changing locations relative to one another are an important determinant of the directions in which they are perceived to move.

In the paradigm that we refer to as induced motion, the movement of one object, such as a luminous rectangle in a dark room, will generally produce an impression that a second stationary object, such as a luminous spot inside the rectangle, is moving in a direction opposite to that of the physical motion of the rectangle. When the rectangle is moving below threshold, it itself is never seen in motion. Therefore, such induced movement cannot entirely be explained on the basis of object-relative displacement. Some explanation is required for the preference on the part of the perceptual system to attribute all of the relative displacement to the spot and none to the rectangle. To deal with this problem, the Gestalt psychologists (e.g., Duncker, 1929) introduced the concept of frame of reference into psychology 
as an analogy to its use in physics. One object was presumed to serve as the reference with respect to which properties of other objects are gauged, motion being one such property. What factors determined which object would become the reference frame was considered an empirical question, and investigation showed that "surroundingness" and size were two of the most important such properties (Oppenheimer, 1934). Implicit in this thinking was that the larger or surrounding object serves as a surrogate of the environment. It implies that the perceptual system "assumes" that the environment or world is stationary. (In other cases, such as the perception of orientation, the frame of reference or world surrogate might be assumed to be upright, so that any object not aligned with it would then be perceived to be tilted.)

Therefore, in the case in which a rectangle is moving below threshold, the two principles referred to above, namely, the central role of object-relative displacement and of frame of reference, can adequately account for induced movement. The perceptual system readily detects the relative displacement and attributes just that much motion to the surrounded spot. But it has been noted that induced movement is also experienced when the rectangle is moving well above subject-relative threshold. This fact introduces two problems for the interpretation above. First, by definition of threshold, the rectangle under these conditions should be seen as a moving object and therefore is not suitable as a stationaryworld surrogate. Second, the relative displacement between the rectangle and spot ought to be fully accounted for by the perceived motion of the rectangle; hence, there is no logical reason to predict induced motion of the spot under these conditions.

Duncker (1929) and later Wallach (1959, 1968) suggested a possible way out of this dilemma with the principle of separation of systems. According to this principle, the attribute of one object (such as motion) is governed more or less exclusively by how it relates to its frame of reference, the one immediately surrounding it; that frame of reference in turn will become an object whose perceived properties are governed by its frame of reference. Thus, the rectangle serves as the frame of reference for the spot, and since there is relative displacement between spot and rectangle, the spot appears to be moving. The rectangle in turn is governed by its relation to the observer, and since it displaces at a rate above threshold, it too appears to be moving. To cite a recent statement of the principle:

\begin{abstract}
Whether or not the rate of motion of the surround is above the threshold for motion based on angular displacement cues seems to matter little. When the motion of the surround is also perceived, the sum of the two experimental motions, the induced motion of the surrounded object and the correctly assigned motion of the surround, may be larger than the relative displacement between the two would warrant; perceiving the motion of the surround does not necessarily diminish the induced motion ... . conditions that cause perceived motion of the surround do not necessarily interfere with the induced motion that is based on configurational change between that surround and the stationary object. (Wallach, Bacon, \& Schulman, 1978)
\end{abstract}

This formulation of the separation-of-systems principle frees induction from its dependence on a stationary world surrogate but introduces a different problem, that of excess motion.

The issue of excess motion is of central concern to us, but first there are other difficulties with the separation-of-systems principle to be discussed. Consider the case in which there is a center spot, an inner rectangle, and an outer rectangle. The separation-of-systems concept suggests that the spot would be perceived in reference to the inner rectangle. Yet, experimentation has shown that a stationary outer rectangle can prevent induction of the spot by motion of the inner rectangle (Brosgole, 1968) and that in situations in which both rectangles are moving in opposite directions, the outer rectangle determines the direction of induced movement of the spot (Brosgole, 1968; Farber, Note 1). These findings indicate that the phenomenal experience of the center spot is a function of the spot's position relative to the outermost frame, a state of affairs that is contrary to the separation-of-systems prediction.

One case that does seem to support the separation-of-systems view is the display in 
which a stationary spot is surrounded by a rectangle moving horizontally, which in turn is surrounded by a circle moving vertically. In this case the spot is sometimes reported to move horizontally but not vertically (Wallach, 1976). However, this effect is difficult to obtain; often the spot is seen as moving vertically or obliquely or as stationary.

As mentioned above, the problem of excess motion is our central concern. Returning to the stimulus conditions of simple induced motion (a stationary spot enclosed by a moving rectangle), we would like to suggest that given veridical perception of the rectangle's motion, there is no reason to expect that the spot will be seen to move. The change of the position of the spot relative to the rectangle should be fully accounted for by the perceived motion of the rectangle. Furthermore, an impression of motion of the spot should be opposed by the absence of change of location of the spot vis-à-vis the observer.

Thus, the solution of perceived motion of the frame and of the spot is irrational. Irrational here means failure to relate the information given in what one might consider to be equivalent to separate propositions when they have a common term, as in logical inference. Thus, if one proposition asserts that there is $X$ amount of relative displacement between $A$ and $B$ and another proposition asserts that there is (the same) $X$ amount of displacement of $B$, then it follows that $A$ is not in motion. Induced motion in this situation implies that more motion is perceived than there is relative displacement-perhaps twice as much. In other words, there is excess phenomenal motion. If Rectangle $\mathrm{A}$ is moving at $3^{\circ}$ per sec and the observer perceives not only this but the spot moving at $3^{\circ}$ per sec in the opposite direction, then there is $6^{\circ}$ per sec of motion perceived with a relative retinal displacement of only $3^{\circ}$ per sec.

We propose the following interpretation as an alternative to the separation-of-systems explanation to encompass not only the case in which the frame is moving below threshold but cases in which it is moving above threshold. There is a strong preference to attribute the relative displacement be- tween a spot and surrounding frame to motion of the spot, and ipso facto, therefore, to perceive the frame as stationary. Thus, the relative displacement between the two that otherwise would be attributed to the motion of the frame is transferred to the spot and thereby subtracted from the frame. To rationalize such phenomenal motion in the spot despite subject-relative information that there is no such motion, observers interpret their eyes as tracking the "moving", spot. Duncker (1929) reported that observers do in fact interpret their eyes as moving even when fixating the stationary spot, and McConkie and Farber (1979) suggest that the retinal drift of the surround could be attributed by the perceptual system to eye movements and thus account for the induced motion of the surrounded object. Such an effect can be considered to be an example of visual capture. It depends on the relative weakness of direct information concerning eye movement or the lack of it in relation to other conflicting evidence. The interpretation of the eyes as tracking the (stationary) spot makes all the more plausible the interpretation of the frame as stationary. The subjective situation is then very much like that which obtains in daily life when we track a moving object in front of a stationary background. The background is then seen as stationary (position constancy). Alternatively to perceiving the frame as stationary, it is also possible to perceive it as moving more slowly than it is (i.e., than it would be perceived when induced motion does not occur). Such an outcome would be based on the tendency to assume the frame is as close to being stationary as is possible under the prevailing conditions. Ipso facto, the spot will then be perceived as moving at a rate less than would be expected were induced movement complete. We will refer to the interpretation offered here as the apportionment hypothesis, by which we mean that the total object-relative displacement caused by the physical movement of the frame will be apportioned by the perceptual system in such a way that no more motion will be perceived than is warranted by that displacement: Either all of the relative displacement will be attributed to motion of the frame or all to the 
spot, or it will be apportioned to spot and frame such that the sum of the two equals the relative displacement.

The experiments to be reported here were undertaken to provide support for the appotionment hypothesis. The main objective of the experiments was to inquire about the perception of the frame, in all cases moving above threshold, during induced-movement trials. It apparently has been assumed by most previous investigators that if the frame is moving above threshold, it will, by definition, be perceived to do so in the inducedmovement paradigm. Therefore, reports concerning the frame as well as reports concerning the spot-with a few exceptions (Gogel, 1977; Gogel \& Koslow, 1971) - have not been obtained.

\section{Preliminary Experiments}

We began with an experiment that entailed the presentation of a series of trials with the rectangular frame set to move at varying speeds. The subject was first to report whether the spot or the frame or both appeared to move and, second, to estimate in inches the extent of such motion. To facilitate accuracy of these estimates, the linear separation between vertical stripes that were inserted in the frame was specified in advance.

\section{Method}

Subjects. The subjects were undergraduate volunteers of both sexes, completely naive about the phenomenon of induced movement. There were 16 subjects in each of the two preliminary experiments to be described here.

Apparatus. A wooden rectangle painted with luminous material served as the moving frame of reference. It was viewed in an otherwise dark room. In one preliminary experiment, this rectangle was 18 in $(45.7 \mathrm{~cm})$ long, 8 in $(20.3 \mathrm{~cm})$ high and contained three vertical stripes $3 / 8$ in $(.95 \mathrm{~cm})$ wide, set 4.5 in $(11.4 \mathrm{~cm})$ apart. The subject sat 43.5 in $(110.5 \mathrm{~cm})$ away so that the visual angle of the rectangle was $23^{\circ}$. The width of rectangle borders was $1 / 2$ in $(1.27 \mathrm{~cm})$. In a second preliminary experiment, a much smaller rectangle was used, namely one half of the size in all dimensions. Moreover, the subject here was 87 in $(221 \mathrm{~cm})$ from the rectangle so that the visual angle of the rectangle was $5.75^{\circ}$, or one quarter of the size subtended by it in the first experiment. The rectangle was moved to the left by means of a chain- drive mechanism. The speed of the rectangle could be varied by means of a transistorized controller. A stationary luminous spot was placed just in front of the rectangle. The spot was near the left edge of the rectangle before the latter was set in motion so that at the end of the trial it was closer to the center of the rectangle. With the head stabilized by a chin rest, subjects viewed binocularly through a large aperture with a sliding panel that opened and closed between trials.

Procedure. Each subject was given trials in both an ascending and descending order, half with the ascending order first and half with the descending order first. The slowest speed, well above the threshold of $.2 \% \mathrm{sec}$ for 2-sec exposure periods according to previous findings (Mack et al., 1975) was $.44^{\circ} / \mathrm{sec}$. For the first experiment, nine other speeds were used, namely, $.67, .89$, $1.11,1.33,1.56,1.83,2.22,2.77$, and $3.44 \% \mathrm{sec}$. For the second experiment, only four of the speeds were used, namely, .44, $1.11,1.83,3.44 \% \mathrm{sec}$.

The moving frame was always exposed for $3 \mathrm{sec}$, which meant that it moved a greater or lesser distance during that period depending on its speed. The subjects were shown the display and told that either the spot or the frame might be moving or both the spot and the frame might be moving. They were asked to open the viewing aperture, to view the luminous spot surrounded by the luminous rectangular frame during the exposure period, to maintain fixation on the spot, and to report what appeared to move and how far in inches.

\section{Results}

In the first of these experiments, which used the larger rectangle, of 320 trials, there were 256 reports of induced movement (or $80 \%$ ). In the second experiment, which used the smaller rectangle, of 128 trials, induced movement occurred on 48 trials (or $371 / 2 \%$ ). There tended to be more induced motion at the slower speeds, particularly in the experiment with the larger frame.

The central question of the research is how the frame is perceived when induced movement does occur. In the experiment with the larger rectangle, of the 256 trials in which induced movement occurred, only the spot appeared to move in 114 trials, or $45 \%$ of the time. In the experiment with the smaller rectangle, of the 48 trials in which induced movement occurred, only the spot appeared to move in 20 trials, or $42 \%$ of the time. Thus, despite the fact that the frame always moved above threshold and in most cases very much above threshold, its motion was not seen on almost half of these trials.

On the remainder of the trials in which induced motion occurred and the frame also appeared to move, we can ask how much the 
frame appeared to move. To answer this question, we compared the average perceived motion in inches of the frame on such trials with the average perceived motion in inches of the frame when induced motion did not occur. If perceived motion in the spot results in a diminished motion in the frame, then the former should be smaller than the latter. The mean of these ratios across all 10 speeds in the experiment with the larger rectangle was .49; in the experiment with the smaller rectangle, it was .82 . Thus, we see that when induced motion occurs, either the frame appears stationary or the extent of its apparent movement is less than when induced motion does not occur.

These results support what one might describe as the weak form of an apportionment principle of induced motion, namely, a tendency of the perceptual system to subtract motion from the frame when attributing the object-relative displacement to the spot. The question to be analyzed next is whether the strong form of the apportionment principle is supported, namely, that there is no significant excess motion at all. However, for a number of reasons this experiment did not lend itself to a decisive answer to this question. Among the difficulties with it were the inaccuracy of judgments and atypical judgments by some subjects based on the use of estimates of displacement in standard linear units; the difficulty for subjects of making both spot and frame judgments when both were perceived to move; and the possibility that when giving both spot and frame estimates, subjects felt constrained to give values that summed to no more than the objectrelative displacement. In designing the experiments to be reported, an attempt was made to eliminate these flaws.

\section{Experiment 1}

\section{Method}

Subjects. The subjects were 10 undergraduate volunteers of both sexes naive about phenomenon of induced movement.

Procedure. The apparatus and physical arrangement were essentially the same as in the preliminary experiments. In this experiment, however, the visual angle subtended by the width of the frame of $5.75^{\circ}$ was achieved by the use of a rectangle measuring 4.5 in across viewed from a distance of 43.5 in. All other di- mensions were reduced proportionately. However, the vertical stripes used in the preliminary experiments were not included.

Judgments of motion were made by the use of two rectangle-and-spot displays that were exact copies of the experimental display. They were mounted one next to the other to one side of the subject. One was used to indicate spot motion. The spot, a small disk, could be slid along a track in the interior of the rectangle from its starting position at one end. Here the rectangle could not be moved. The other display was used to indicate frame motion. The frame could be moved with respect to the spot, which in this display remained stationary. Distances could be read off from a ruler in back of the displays not visible to the subject. The subjects understood that if only the spot appeared to move, they were to indicate the distance it appeared to move by sliding the spot the correct distance on the appropriate display; if only the rectangle appeared to move, they were to indicate the distance it appeared to move by sliding the rectangle along appropriately on the other display. If both spot and rectangle appeared to move, they were to indicate the apparent displacement of only one or the other, as the instructions given later will make clear. These judgments were made in the light that was turned on immediately following observation of the experimental display in the dark. This method of direct-comparison settings eliminated the difficulty with the preliminary experiment of obtaining judgments in terms of estimating linear units.

The subjects were told that on each trial in the dark, they would see either the spot move, the rectangle move, or possibly both move. They were to maintain fixation on the spot at all times and to note what they perceived to be moving. They were shown the comparison displays, and it was explained how they were to use these to indicate the extent of displacement of either object. Immediately after the exposure period, they were to say what had appeared to move. They were further told that on trials on which they perceived both spot and rectangle moving, they would have to judge the displacement of one or the other and that they would be told which one directly after their verbal response of "both." The selection of the required matching on such both-response trials was based on a prerandomized sequence. This method of sampling for bothresponse trials eliminated the other two difficulties with the preliminary experiments referred to above that stemmed from the requirement of judging both spot and rectangle on any one trial.

On one third of the experimental trials, the spot was moved rather than the rectangle. The major purpose of these trials was to provide a baseline of perceived displacement against which to assess the magnitude of perceived displacement on induced-movement trials. There were 8 such trials and 16 induced-movement trials. Four speeds were used, namely, Speeds 1, 2, 4, and 5 of the first preliminary experiment. These were, respectively, $.44, .67,1.11$, and $1.33^{\circ}$ per sec. Since the display was visible for $3 \mathrm{sec}$, the actual displacements were, respectively, $1,1.6,2.5$, and 3 in $(2.54,4.1,6.3$, and $7.6 \mathrm{~cm}$, respectively). There were 2 trials at each of these speeds on the spot-motion trials and 4 on the induced-motion trials. The order of the 24 trials was 
random with the one constraint that one spot-motion trial and two rectangle-motion trials of the same speed were clustered together. The purpose of this clustering was to ensure that any slight change in the setting of the dial of the variable speed controller would not affect the speed differentially for spot-motion and rectanglemotion trials of the same speed. The dial could be set and left unchanged for the 3 trials in each such cluster.

\section{Results}

Induced movement was experienced on 110 of 160 rectangle-motion trials, or $69 \%$ of the time. Of these, only the spot was reported to be moving on 75 trials, or $68 \%$ of all induced-movement cases. On the remaining 35 trials, or $32 \%$ of the time, both spot and rectangle were seen as moving. Needless to say, the spot was always perceived to be moving on the spot-motion trials except for 1 of these 80 trials in which the rectangle was reported to have moved.

We thus confirmed and in fact found even more prevalent here the finding of the preliminary experiment that the frame often appears stationary when it is moving above threshold, provided induced movement of the spot occurs. The greater incidence of such cases in the present as compared to the preliminary experiments may be based on the fact that the four speeds used were among the slower ones included in those ex- periments. When induced movement occurred at the slower speeds, it tended to be of this kind, that is, with the frame appearing to be stationary, whereas when it occurred at faster speeds, there were proportionately more both reports. In the present experiment, for example, of the 38 instances of induced motion at the slowest speed, 33 (or $87 \%$ ) were of this kind, whereas of the 13 instances of induced movement at the fastest speed, only $5(38 \%)$ were of this kind. In passing, it is worth noting another fact revealed in the previous sentence, namely, that induced movement of any kind occurred more frequently at the slower speeds, and this was true in the preliminary experiment as well.

As to the extent of motion perceived on trials when both spot and rectangle were reported moving, we will consider first those trials on which a judgment concerning the rectangle only was requested. The average displacements perceived are given in row A of Table 1 , and the average displacements judged for these speeds when the spot was moved are given in row $C$. These latter values can serve as an index of the magnitude of the frame-motion perception that we should expect based on the relative displacement between spot and frame. Alternately, we can use as such an index the per-

Table 1

Mean Comparison Matches of Perceived Displacement in Inches in Experiment 1

\begin{tabular}{|c|c|c|c|c|c|c|c|c|c|c|c|c|}
\hline \multirow[b]{2}{*}{ Response category } & \multicolumn{3}{|c|}{$\begin{array}{l}.44 \% \mathrm{sec}^{\mathrm{a}} \\
1.0 \text { in. }\end{array}$} & \multicolumn{3}{|c|}{$\begin{array}{l}.67^{\circ} / \mathrm{sec}^{\mathrm{a}} \\
1.6 \mathrm{in}^{\mathrm{b}}\end{array}$} & \multicolumn{3}{|c|}{$\begin{array}{c}1.11 \% / \mathrm{sec}^{\mathrm{a}} \\
2.5 \text { in. }^{\mathrm{b}}\end{array}$} & \multicolumn{3}{|c|}{$\begin{array}{c}1.33 \% \mathrm{sec}^{\mathrm{a}} \\
3.0 \mathrm{in}^{\mathrm{b}}\end{array}$} \\
\hline & $M$ & $S D$ & $n$ & $M$ & $S D$ & $n$ & $M$ & $S D$ & $n$ & $M$ & $S D$ & $n$ \\
\hline $\begin{array}{l}\text { A. Frame matches on "both } \\
\text { response" trials } \\
\text { B. Frame matches when induced }\end{array}$ & .5 & 0 & 2 & .81 & .39 & 6 & 1.25 & .68 & 9 & 1.0 & 1.19 & 4 \\
\hline movement did not occur & 1.18 & .18 & 2 & 1.69 & .35 & 6 & 2.45 & .34 & 15 & 2.88 & .19 & 27 \\
\hline $\begin{array}{l}\text { C. Spot matches on spot- } \\
\text { displacement trials }\end{array}$ & 1.0 & .31 & $19^{c}$ & 1.80 & .17 & 20 & 2.68 & .31 & 20 & 3.24 & .15 & 20 \\
\hline $\begin{array}{l}\text { D. Spot matches on induced } \\
\text { movement trials (frame } \\
\text { seen as stationary) }\end{array}$ & 1.26 & .26 & 33 & 1.81 & .21 & 27 & 2.74 & .40 & 11 & 3.18 & .12 & 5 \\
\hline $\begin{array}{l}\text { E. Spot matches on "both } \\
\text { response" trials }\end{array}$ & .75 & .18 & 3 & 1.25 & - & 1 & 1.85 & .78 & 5 & 1.75 & .95 & 4 \\
\hline
\end{tabular}

Note. $1 \mathrm{in}=2.54 \mathrm{~cm}$.

a Angular velocity.

b Objective displacement.

c One subject on one trial reported the frame as moving. 
ceived displacement of the rectangle when no induced movement occurred (row B), or we can use the objective distances traversed by the rectangle. Whichever of these indices of motion are used as a measure of the magnitude of the perceived motion of the rectangle that should be expected to occur, it is perfectly clear that when induced motion occurs and the frame is perceived to be moving, its motion is seen to be substantially less than would be the case were no induced motion occurring. In fact, in these cases the motion of the frame perceived is half or less than half of the motion we should otherwise expect.

It is evident, therefore, that induced motion is accompanied by a subtraction of phenomenal motion from the frame, either entirely, in the majority of cases, or partially, in cases in which both spot and rectangle are seen to move. Thus, there is considerable evidence in support of the weak version of the apportionment hypothesis.

To test the strong version of the hypothesis, that there is in effect no significant amount of excess motion, we require the average of the sums of both perceived spot and frame motion at each speed. For cases in which induced movement occurs and the frame is seen as stationary, there is no difficulty. The sum for any trial is the judged displacement of the spot. But for cases in which both spot and frame are reported to be moving, we have only one or the other judgment. Since we randomly sample perceived spot motion on some trials and rectangle motion on other trials, we presume that were we to add a spot judgment from one trial to a rectangle judgment from another trial at the same speed, we would arrive at an estimate of what the sum would have been had we obtained both judgments on the same trial. This means that we can arrive at an estimate of the mean of sums of both-response trials for a given speed simply by averaging all the single judgments multiplied by two. Finally, we can take these values together with those trials on which the spot only appeared to move and compute the overall average. These are $1.26(N=38), 1.80(N=34), 2.85(N=$ $25)$, and $2.92(N=13)$ for the four speeds, respectively. If we compare these with the average perceived displacement of the spot on the spot-motion trials (row C), it can be seen by inspection that the values for each speed are fairly similar. The overall average of these values is 2.20 for sums of induced motion and 2.19 for perceived motion of the moving spot, and these clearly are not significantly different. ${ }^{1}$ Thus, in this experiment the strong version of the apportionment hypothesis is supported.

It is worth noting that if a separation-ofsystems effect occurred and were complete, whenever induced motion occurred, the spot should appear to move as much as it displaces relative to the rectangle and the rectangle as much as it displaces relative to the spot and to the subject. Using the spotmotion trials as the best index of these values for the spot (row C) and the induced motion trials in which only the rectangle appeared to move as the best index of these values for the rectangle (row $B$ ) and adding the two for each speed, we get, respectively, $2.18,3.49,5.13$, and 6.12 . One can see just how much greater these values are than the average sums of both actually obtained.

${ }^{1}$ The reader may think that another test, simpler than the one used, is available to test the hypothesis, namely, adding together the means of row A (frame matches on both-response trials) and row $\mathrm{E}$ (spot matches on both-response trials) for each speed and comparing these sums to the objective displacement or perceived frame movement in the absence of induced motion (row B). However, there are reasons for not using this test. First, it excludes the data for trials in which induced motion is complete, that is, in which only the dot is seen as moving and the frame is reported as stationary (row D). These trials represent absence of any excess motion and certainly should be included in a test for apportionment. These are instances in which all the object-relative displacement is attributed to motion of the spot. Second, neither the objective nor the perceived displacement of the frame is the best indicator of what responses to expect, given the absence of excess motion. The perceived motion of the spot on spot-displacement trials (row $C$ ) is, in our opinion, the least ambiguous indicator of the amount of perceived motion that can be expected for the given magnitudes of object-relative displacement because it precludes any motion illusions (only the object that is physically in motion is seen to move, viz., the spot), and there are an adequate number of cases and no sampling problem, since, unlike the case for row $B$ or $D$, we obtain a response for each of the 20 trials given at each speed. 


\section{Experiment 2}

There were two major changes in the procedure of this experiment. First, instead of a matching technique, the method of magnitude estimation was used. Second, instead of allowing the subject to report spontaneously whether the spot, rectangle, or both appeared to move, a sampling method was used. The experimenter requested that the subject report about either the spot or the rectangle; that is, directly following the presentation of each induced movement trial the experimenter either said "spot" or "rectangle," and that was the signal for the subject to respond only about the spot or rectangle in terms of the estimated magnitude of its motion. This method assured that for each subject and for each speed, an equal number of judgments would be secured about the spot's displacement and the rectangle's displacement. This of course was not the case in Experiment 1 even for both responses in which the experimenter did call for either a spot or a rectangle judgment and to that extent is a minor flaw in that experiment as far as an assessment of the data is concerned.

\section{Method}

Subjects. The subjects were 16 undergraduate volunteers of both sexes naive about phenomenon of induced movement.

Procedure. The apparatus and physical arrangement was the same as in Experiment 1. We used a 4.5-in wide rectangle with three vertical stripes and the same four speeds.

The procedure was as follows: Prior to each of 16 induced-movement trials given to each subject (4 at each of the four speeds), a standard trial was given in which only the spot moved. The speed of the spot on these latter trials was the same as the speed of the rectangle on the induced-movement trial that followed it and with which it was paired. The order of these 16 paired trials was randomized for each subject. The subject understood that the standard trial provided the baseline displacement against which to gauge the displacement in whatever the experimenter required to be judged on the trial that followed it. The instructions to the subject were as follows:

Your task will be to tell us how far either this rectangle or this small spot appears to move. We will show you the spot moving a given distance. You will think of that distance as having a numerical value of 10. Soon after this you will receive a trial, at the end of which we will ask you to report on the motion of either the spot or the rectangular frame. Your response will be a number that represents the distance the object appeared to move, as compared with the standard distance 10. For example, if you are asked to report on the distance the frame moved, and you think the frame moved twice the distance of the spot on the standard presentation, you would report a value of 20 . If the frame did not appear to move at all, you would report 0 . You may choose any number that indicates what you saw. A report of 5 would indicate the object moved one half the standard distance, 10 that it moved an amount equal the standard, and 15 an amount $1 \frac{1}{2}$ times the standard distance. These are intended as examples to make the use of the number values clear. Once we begin it is important that you always fixate the spot.

Several practice pairs of trials were given to make sure that the task and the instructions were understood, and on some of these practice trials the spot rather than the rectangle was actually moved. It is important to note that the subjects did not know whether they would have to report on the motion of the spot or the frame until the trial ended. Had we informed them in advance, it is possible that this would have led to a focus of attention that would have affected the percept itself.

\section{Results}

Of the 128 trials requiring a report about the spot ( 8 trials per subject $\times 16$ subjects), 56 yielded values of 0 and 72 greater than 0 . Thus, induced movement occurred $56 \%$ of the time. Once again, there was clear evidence that the incidence of induced movement was inversely related to the speed of the rectangle. For example, at the slowest speed, it occurred on 25 of the 32 trials, whereas at the fastest speed it occurred on only 7 of 32 trials.

Of the 128 trials requiring a report about the rectangle, 47 yielded a value of 0 and 81 a value greater than 0 . Thus, in this experiment too, the frame was often reported to be stationary ( $37 \%$ of the time) despite the fact that it moved well above threshold on all trials. In fact, in this experiment, the percentage of induced-movement trials in which the frame appeared to be stationary is much higher than $37 \%$. That is because on many of the trials in which a frame report was required, induced motion of the spot would not have occurred. We can estimate the probable number of induced-movement cases from the trials when a spot report was required and on which induced movement occurred, namely, 72. Assuming roughly the 
same number of cases for frame-judgment trials, we can then infer that in 47 out of 72 cases of induced movement, or $65 \%$ of the time, the rectangle appeared to be stationary. This value is similar to that obtained in Experiment 1, namely $68 \%$. Thus, here again we find strong evidence of a subtraction process in which all the motion of the frame is transferred to the spot. Thus, there is certainly strong evidence in the experiment for the weak version of the apportionment hypothesis.

To test the strong version of this hypothesis, we must first consider the rationale behind the procedure of this experiment. If, on a given induced-motion trial, both the spot and the rectangle appear to move by as much as the spot appears to move on the standard trial, then whether we call for a spot or rectangle response, the subject should give a report of 10 . This would be in accord with the separation-of-systems hypothesis. The average report would therefore be 10 . Or if the rectangle appears to move by as much as the spot on the standard trial and the spot appears to move but less so, then if we call for a rectangle response, the report should be 10 , but if we call for a spot response, the report should be some value less than 10 but greater than zero. This would be in accord with a separation-of-systems hypothesis according to which induced movement is less than complete. The average report would, therefore, be some value between 5 and 10 . But if the apportionment hypothesis is correct, then the average report should be 5 . Either the spot or the frame should appear to move as much as the spot in the standard trial and thus be assigned a value of 10 , and the other object, frame or spot, respectively, should appear to be stationary. But by our sampling method, we should expect a report of 10 or 0 equally often, the average thus being 5 . Or the objectrelative displacement will lead to a distribution of perceived motion of the spot and frame that sums to 10 , in which case, again, by our sampling procedure, the average should be 5 .

The overall mean of the means of the 16 trials for all 16 subjects was $5.2(S D=$ 1.22 ), a value that obviously does not differ significantly from a true mean of 5 . For the eight trials per subject on which a report of the spot's motion was required, the overall mean of the means for all 16 subjects was 4.7 $(S D=1.63)$, and the corresponding overall mean of trials on which a report of the rectangle's motion was required was 5.7 $(S D=2.1)$.

However, it might be argued that for the many trials on which induced movement does not occur, the average report should be 5 , since the spot report would be 0 , and the frame report would be expected to be roughly 10 . Therefore, the separation-ofsystems prediction would not be an average of 10 for all trials but some lower value. Since in fact induced movement occurred on $56 \%$ of all trials, we can correct the prediction based on separation of systems as follows: For trials on which induced movement occurred, the predicted average report is 10 , and this must be weighted by a factor of .56; for trials on which induced movement did not occur, the predicted average report is 5 , and this must be weighted by a factor of .44 . The overall average therefore must be 7.8 . However, it might be further argued that separation of systems need not be complete on trials when induced movement does occur. Thus, the prediction from this modified hypothesis is simply that some excess motion will occur. But we have found that the average report of 5.2 does not differ from the strict-apportionment hypothesis prediction, which means that no significant excess motion at all was perceived.

\section{Discussion}

The most striking finding in these experiments is the fact that there were so many cases in which induced movement occurred and in which the rectangle, moving at a speed above threshold, was perceived to be stationary. When induced movement did not occur, the rectangle's motion was always perceived, and the extent of its motion was perceived more or less accurately, judging from the average values assigned by subjects in the experiments reported here. That the tendency to perceive the rectangle as stationary when induced motion of the 
spot occurs is based on a process of subtraction from the total object-relative displacement is further substantiated by a control experiment not yet reported. In this control, subjects judged the displacement of the rectangle in inches when the spot was not present at all. Of 64 trials, representing equally often the four speeds used in these experiments, only 1 produced a report of zero. The conclusion seems inescapable, therefore, that the tendency of the perceptual system to attribute the relative displacement between spot and rectangle to a motion of the spot is the cause of this subtraction effect. This finding, together with the quantitative analysis of the sum of motion perceived in the spot and frame in the experiments reported, strongly supports the apportionment hypothesis. There would thus seem to be no need to invoke the concept of separation of systems to explain induced motion in the case in which the inducing object is moving above threshold.

Certain other facts fit this interpretation. For example, as noted earlier, induced movement is difficult to achieve when a stationary frame surrounds the moving one, or, what amounts to the same thing, it is difficult to achieve under daylight conditions in which there are stationary structures surrounding the moving one. For in these cases, it is not possible to misperceive or underestimate the motion of the moving frame. In other words, when visible stationary objects are present outside the moving frame, the object-relative displacement thereby provided will surely lead to the veridical perception of the moving frame's motion. Therefore, the subtraction process necessary for induced motion cannot occur.

Then there is the case described earlier of two frames surrounding a stationary spot and displacing simultaneously in opposite directions. Here the spot generally appears to move in a direction opposite to that of the outermost frame (Brosgole, 1968; Farber, Note 1). This is in direct contradiction to the separation-of-systems prediction according to which the relation of the induced object to the immediately surrounding frame is paramount. However, according to the interpretation suggested here, the motion of the inner frame would not be underestimated in this case-it could hardly be seen as stationary. But the motion of the outer frame could be. In that event we should predict the obtained outcome, namely, that the relative displacement between the outer frame and the spot will lead to induced motion of the spot in a direction opposite to the motion of the outer frame. Incidentally, we should also predict some induced motion of the inner frame so that its phenomenal speed would be overestimated, a fact not mentioned in the investigations cited.

We view the phenomenon of induced movement as based on a process of problem solving. The proximal input is registered and interpreted in terms of what external event is most plausibly producing that input (Helmholtz, 1866). The perceptual system is particularly sensitive to stimulus relationships and, we would go so far as to say, always tends to achieve a solution that will fully account for such relationally given information. In the case of motion, that information is object-relative displacement. Ordinarily, when an object moves, it is in relation to a surrounding stationary environment, and subject-relative information-based on the changing angular direction of the objectcorroborates that of the object-relative displacement to the effect that the object is moving. In the case of induced movement, however, there is a conflict between the two sources of information, and apparently, up to a point, object-relative information is dominant. But inasmuch as the object-relative displacement would also be intelligently accounted for simply by perceiving the frame as moving without any induced motion occurring, we must also maintain that there is a preference on the part of the perceptual system to interpret the surrounding or larger object as stationary or moving more slowly than it is. Finally, since the eyes are fixating the stationary object, for induced motion to be rationalized, that is, to avoid contradiction, it appears as if they are tracking the phenomenally moving spot.

These last points about conflict and information concerning angular direction are relevant to the finding reported here and elsewhere (Brosgole, 1968; Duncker, 1929) that induced movement occurs more otten when the speed of the inducing object is slow rather than fast. It is not clear why speed should be relevant from the standpoint of 
the separation-of-systems doctrine, or for that matter, from any other view of induced movement thus far propounded. Although we cannot fully explain this finding, it stands to reason that the more rapid the displacement of the inducing object, the better the information that it is changing its angular direction. Since our argument is that for induced motion to occur, the inducing object's motion must either not be perceived at all or it must be underestimated, it follows that the better the information about its true motion, the less likely is the occurrence of induced movement. Moreover, faster speeds of the inducing object logically require faster perceived speed of the induced object, and such rapid phenomenal speed of the induced object is more in conflict with the information that it is stationary than slow phenomenal speed would be.

It might be argued that there are reasons why complete separation of systems leading to "complete" induced motion of the spot should not be expected to occur. As noted above, information from changing angular direction (which is based on information from the eye-retina system) is available to the effect that the spot is stationary. Thus, a conflict exists, with one determinant suggesting that the spot is in motion and another that it is not. As a result, the spot may only appear to move partially. This formulation changes the meaning of separation of systems as first put forth by Duncker (1929) because it implies that the frame does not completely insulate the spot from the observer as frame of reference. In any event one might attempt to maintain a modified separation-of-systems hypothesis by predicting only partial induced movement for the reason given. But according to this analysis, the frames' motion should always be perceived veridically, since there is no conflicting information concerning it. Our finding that it is not and that when it is perceived to be stationary, the induced motion of the spot is indeed complete, argues against this interpretation. Moreover, our findings are not only contrary to predictions based on complete separation of systems but are contrary to predictions based on any separation of systems. That is because we find no evidence for any significant excess motion in the inducedmotion paradigm.
There are, however, certain facts and findings about induced motion that contradict or do not seem to follow from the interpretation we have offered here. In the only other experiment we know about in which quantitative data were obtained concerning perceived spot and frame motion (Gogel \& Koslow, 1971), a result contrary to ours indicating an appreciable amount of excess motion was reported. In fact, it would appear that the results approximate those that one would predict from the standpoint of the hypothesis of a complete separation of systems. We are at a loss to explain this contradiction except for the fact that in that study a 30-sec exposure period was used in which the moving frame reversed its direction several times. In that long a period, it is entirely probable that perceptual reversals and shifts of attention occurred, so that there may well have been moments when only the spot was seen in motion and other moments when only the frame was seen in motion. The observers may then afterward have assumed that each moved throughout the $30-\mathrm{sec}$ period and thus arrived at the estimate in inches required.

Then there is the fact that some impression of motion may be induced in a stationary object when the inducing object consists of a series of contours, for example, stripes, that move across it even when the motion of the inducing object is not misperceived. A related example is that of induced rotary motion in which a stationary disk with a series of contours at right angles to the edge is surrounded by another concentric rotating disk with such contours adjacent to those on the inner disk. One has the impression that the inner disk is rotating in a direction opposite to that of the outer one. It is unlikely that the rotation of the outer disk is misperceived. However, in these examples, the motion is paradoxical; that is, it is phenomenally nonspatial. The induced object does not appear to change its location in space. This sensation of motion may thus be of a different character and may result directly from some motion-detection mechanism sensitive to relative displacement of adjacent contours. Such an effect might conceivably have occurred in our experiments, particularly when we used a rectangle containing vertical stripes. If so, 
our failure to find any significant excess motion is all the more noteworthy.

Another finding we cannot explain is one recently reported by Gogel (1977) that induced motion can occur simultaneously in opposite directions for two stationary spots, each surrounded by rectangles moving out of phase with one another. We would expect, consistent with our hypothesis, that for any induced motion to occur, one frame would appear to be stationary or moving slower than it is and that both spots would then appear to move together in a direction opposite to the actual motion of that frame. The other frame should appear to move rapidly toward or away from that frame, since all or most of the relative displacement between frames would be attributed to it. This is precisely what Brosgole (1968) found in an earlier version of this kind of experiment for those subjects who experienced induced motion. The chief difference in conditions between the two experiments is that Brosgole's rectangles were one above the other with the spots vertically aligned, whereas Gogel's rectangles were side by side with spots therefore also horizontally separated from one another by an appreciable visual angle. Presumably the observers at any moment must have been directing their gaze to one spot and frame so that the other would be seen only peripherally. A similar effect has been reported by Nakayama and Tyler (1978) in which stationary lines surrounded by lines moving in counter phase appear simultaneously to move in directions opposite to one another. This effect is strictly limited to certain minimum and maximum velocities. Perhaps, therefore, these opposite induced-motion effects are phenomenally of the nonspatial variety referred to earlier and depend on the activation of a motion-detection mechanism driven by relative displacement of contours. In any event, further research with these kinds of displays will be necessary to clear up this seeming contradiction.

Then there are certain effects that can be thought of as entailing induced movement in which the object in question is in fact physically moving. The perceived direction of this motion is, however, affected by the motion of other objects in the field. Thus, one might argue that this perceived direction is the result of two vectors: the object's actual motion and the induced motion imparted by the motion of other objects or frames. For example, Wallach et al. (1978) have reported experiments in which a display of vertical lines moves horizontally across a spot that moves vertically. The spot appears to move obliquely. This effect can be understood if one assumes that the spot undergoes induced motion along a horizontal axis. The oblique motion is then the result of induced horizontal motion and objective vertical motion. We have observed this kind of effect and have the impression that as with simple induced motion, it is based at least in part on subtraction from the motion of the frame. At slow speeds the frame appears to be stationary. However, at faster speeds, when the displacement of the lines and the spot are equal, the spot appears to move at approximately a $45^{\circ}$ angle, which is what one would predict if the induced movement were complete. According to the apportionment hypothesis, that should only occur when the frame appears to be stationary, but the fact is that this effect occurs when the frame clearly is seen to be moving. Thus, it may well be that excess motion occurs in this paradigm and, if so, it leaves us with the problem of why it does here but not in the simple induced-motion paradigm.

Some brief reference to induced movement of the self is warranted, a phenomenon known to perception investigators and discussed in textbooks but neglected in the laboratory until recently (Brandt, Dichgans, \& Koenig, 1973; Duncker, 1929; Johansson, 1977; Lee, 1974; Lishman \& Lee, 1973; Rock, 1968; Wong \& Frost, 1978). This effect requires special conditions, chief among which is that the observer be or appear to be surrounded by or enclosed within the moving framework. We are assuming that this effect did not occur in the experiments described here. None of our subjects reported it, nor did any of the authors or others in our laboratory experience it under our experimental conditions. When the effect does occur, the inducing frame is experienced as stationary, although this may be preceded by a stage in which it appears to be moving more slowly than is warranted and at which time the observers 
experience themselves as moving more slowly than at the final stage. This is a paradigm that illustrates most clearly the tendency of the perceptual system to interpret the frame as a surrogate of the stationary environment. It also illustrates the apportionment process at work, although here the relative displacement that matters is between self and frame. Recent experiments have shown how the sum of perceived frame plus self-movement is constant and equal to the angular change of the frame per unit time (Wong \& Frost, 1978), a fact that we have independently observed in our laboratory.

If one places a stationary spot just in front of the moving frame that surrounds the observer, conditions for both induced objectmotion and induced self-motion obtain. We have found that the spot is then perceived in a manner consistent with the way the self is perceived; prior to the onset of induced movement of the self, the stripes appear to be moving and the spot appears to be stationary (although if the frame consists of stripes, a paradoxical, nonspatial sensation of spot motion may be experienced). After the onset, when the stripes appear to be stationary, the spot appears to be moving. In this situation, it is as if self and spot were yoked together such that however one is perceived, so is the other. Thus, the outcome always appears to be a rational solution. Interestingly enough, induced object motion is here not one of changing angular direction with respect to the observer as in the simple induced-movement paradigm. The spot always appears to be straight ahead of the observer.

\section{Reference Note}

1. Farber, J. M. Peripheral dominance in dual-surround induced motion. Paper presented at the meeting of the Eastern Psychological Association, Philadelphia, Pa., April 1979.

\section{References}

Brosgole, L. Analysis of induced motion. Acta Psychologica, 1968, 28, 1-44.

Brandt, T., Dichgans, J., \& Koenig, E. Differential ef- fects of central versus peripheral vision on egocentric and exocentric motion perception. Experimental Brain Research, 1973, 16, 476-491.

Duncker, K. Uber induzierte Bewengung. Psychologische Forschung, 1929, 12, 180-259. (Translated and condensed in W. Ellis [Ed.], Source book of gestalt psychology, Humanities Press, Inc. Selection 12, 1950).

Gogel, W. C. Independent motion induction in separated portions of the visual field. Bulletin of the Psychonomic Society, 1977, 10, 408-411.

Gogel, W. C., \& Koslow, M. The effect of perceived distance on induced movement. Perception \& Psychophysics, 1971, 10, 142-146.

Helmholtz, H. von. Handbuch der Physiologischen Optik (Vol. 3). Leipzig, Germany: Voss, 1866.

Johansson, G. Configuration in event perception. Uppsala, Sweden: Almkvist und Wiksell, 1950.

Johansson, G. Studies on visual perception of locomotion. Perception, 1977, 6, 365-376.

Lee, D. L. Visual information during locomotion. In R. B. Macleod \& H. L. Pick, Jr. (Eds.), Studies in perception: Essays in honor of James J. Gibson. Ithaca, N.Y.: Cornell University Press, 1974.

Lishman, J. R., \& Lee, D. N. The autonomy of visual kinesthesis. Perception, 1973, 2, 287-294.

Mack, A., Fisher, C. B., \& Fendrich, R. A reexamination of two-point induced movement. Perception \& Psychophysics, 1975, 17, 273-276.

McConkie, A., \& Farber, J. Relation between perceived depth and perceived motion in uniform flow fields. Journal of Experimental Psychology: Human Perception and Performance, 1979, 5, 501508.

Nakayama, K., \& Tyler, C. W. Relative motion induced between stationary lines. Vision Research, 1978, 18, 1663-1668.

Oppenheimer, I. Optische Versuche uber Ruhe und Bewegung. Psychologische Forschung, 1934, 20, $1-46$.

Rock $I$. The basis of position constancy during passive movement of the observer. American Journal of Psychology, 1968, 81, 262-265.

Wallach, H. The perception of motion. Scientific American, 1959, 20I, 56-60.

Wallach, H. Information discrepancy as a basis of perceptual adaptation. In $\mathrm{S}$. J. Freedman (Ed.), The neuro-psychology of spatially oriented behavior. Homewood, Ill.: Dorsey Press, 1968.

Wallach, H. On perception. New York: Quadrangle/ The New York Times Book Co., 1976.

Wallach, H., Bacon, J., \& Schulman, P. Adaptation in motion perception: Alteration of induced motion. Perception \& Psychophysics, 1978, 24, 509-514.

Wong, S. C. P., \& Frost, B. J. Subjective motion and acceleration induced by movement of observers' entire visual field. Perception \& Psychophysics, 1978, $24,115-120$.

Received August 27, 1979 